Bull. Korean Math. Soc. 52 (2015), No. 1, pp. 183-200

http://dx.doi.org/10.4134/BKMS.2015.52.1.183

\title{
DIRECTIONAL ASSOCIATED CURVES OF A NULL CURVE IN MINKOWSKI 3-SPACE
}

\author{
JinhuA QIAN AND Young Ho Kim
}

\begin{abstract}
In this paper, we define the directional associated curve and the self-associated curve of a null curve in Minkowski 3-space. We study the properties and relations between the null curve, its directional associated curve and its self-associated curve. At the same time, by solving certain differential equations, we get the explicit representations of some null curves.
\end{abstract}

\section{Introduction}

In the history of differential geometry, the theory of the associated curve of a given curve has been one of interesting topics. Many geometers have investigated this problem from various viewpoints: For instance, in Euclidean 3 -space, the Bertrand partner and the Mannheim partner curves are two important kinds of associated curves. They are characterized by the curvature and the torsion, namely, a Bertrand curve satisfies $\lambda \kappa+\mu \tau=1$ and a Mannheim curve has $\kappa=\lambda\left(\kappa^{2}+\tau^{2}\right)$, where $\kappa$ is the curvature and $\tau$ the torsion.

In Minkowski 3-space, there are three kinds of typical curves, that is, spacelike, time-like and null (light-like) curves. Among them, a null curve is quite different from other types of curves. Motivated by the idea about partner curves, we naturally consider the associated curves of a null curve in Minkowski 3 -space.

In [4], the authors defined the $W$-direction curve $\tilde{r}$ of a null curve $r$ in such a way that the tangent vector field of $\tilde{r}$ is $W$, where $W$ is a vector field along the given null curve. In this case, the curve $r$ is called the $W$-direction donor curve of $\tilde{r}$.

Received December 10, 2013.

2010 Mathematics Subject Classification. Primary 53A04, 53B30.

Key words and phrases. Minkowski space, null curve, null curvature, directional associated curve, self-associated curve.

The first author was supported by NSFC (No. 11371080). The second author was supported by Basic Science Research Program through the National Research Foundation of Korea (NRF) funded by the Ministry of Education, Science and Technology (2012R1A1A2042298) and supported by Kyungpook National University Research Fund, 2012 . 
In this paper, we define the directional associated curves of a null curve, and introduce the notion of the self-associated curve of a null curve. Using the representation formulas of cone curves introduced in [8], we study the properties of a null curve and its directional associated curves in Minkowski 3-space.

In Section 2, we will review some basic facts for null curves and non-null curves in Minkowski 3-space (see [1]-[8]). In Sections 3-5, we discuss some specific directional associated curve of a null curve. In Section 6, we study the relations between the null curve and its self-associated curve.

All geometric objects under consideration are smooth and curves are regular unless otherwise stated.

\section{Preliminaries}

In this section, we review some basic facts for null curves and non-null curves in Minkowski 3-space.

\subsection{Vector product in Minkowski 3-space}

Let $E_{1}^{3}$ be a Minkowski 3-space with natural Lorentzian metric

$$
\langle\cdot, \cdot\rangle=d x_{1}^{2}+d x_{2}^{2}-d x_{3}^{2}
$$

in terms of the natural coordinate system $\left(x_{1}, x_{2}, x_{3}\right)$.

Let $a=\left(a_{1}, a_{2}, a_{3}\right), b=\left(b_{1}, b_{2}, b_{3}\right)$ and $c=\left(c_{1}, c_{2}, c_{3}\right)$ be vectors in $E_{1}^{3}$. Then their scalar product is given by

$$
\langle a, b\rangle=a_{1} b_{1}+a_{2} b_{2}-a_{3} b_{3},
$$

and the exterior product by

$$
a \times b=\left|\begin{array}{lll}
e_{1} & e_{2} & e_{3} \\
a_{1} & a_{2} & a_{3} \\
b_{1} & b_{2} & b_{3}
\end{array}\right|=\left(\left|\begin{array}{ll}
a_{2} & a_{3} \\
b_{2} & b_{3}
\end{array}\right|,\left|\begin{array}{ll}
a_{3} & a_{1} \\
b_{3} & b_{1}
\end{array}\right|,-\left|\begin{array}{ll}
a_{1} & a_{2} \\
b_{1} & b_{2}
\end{array}\right|\right),
$$

where $\left\{e_{1}, e_{2}, e_{3}\right\}$ is an orthonormal basis in $E_{1}^{3}$. One can have

$$
e_{1} \times e_{2}=-e_{3}, \quad e_{2} \times e_{3}=e_{1}, \quad e_{3} \times e_{1}=e_{2} .
$$

On the other hand, the mixed product is given by

$$
\langle a \times b, c\rangle=\left|\begin{array}{ccc}
a_{1} & a_{2} & a_{3} \\
b_{1} & b_{2} & b_{3} \\
c_{1} & c_{2} & c_{3}
\end{array}\right|
$$

and the double exterior product is then obtained as

$$
(a \times b) \times c=\langle b, c\rangle a-\langle a, c\rangle b .
$$

A vector $v \in E_{1}^{3}$ is said to be space-like if $\langle v, v\rangle>0$ or $v=0$; time-like if $\langle v, v\rangle<0$; null (light-like) if $\langle v, v\rangle=0$, respectively.

Remark 2.1. Hereafter, we assume that a null geodesic in $E_{1}^{3}$ is not regarded as a null curve. 


\subsection{Frenet formulas of null curves in $E_{1}^{3}$}

Let $r(s)$ be a null curve with parameter $s$ in $E_{1}^{3}$. If $\left\langle r^{\prime \prime}(s), r^{\prime \prime}(s)\right\rangle \neq 0$, we can take $\tilde{s}$ appropriately in such a way that $\left\langle r^{\prime \prime}(\tilde{s}), r^{\prime \prime}(\tilde{s})\right\rangle=1$. In this case, the parameter $\tilde{s}$ is called the null arc length parameter. For a null curve $r=r(s)$ with null arc length parameter $s$, there exists a unique frame field $\{x, \alpha, y\}$ such that

$$
\left\{\begin{array}{l}
r^{\prime}(s)=x(s) \\
x^{\prime}(s)=\alpha(s) \\
\alpha^{\prime}(s)=\kappa(s) x(s)-y(s), \\
y^{\prime}(s)=-\kappa(s) \alpha(s)
\end{array}\right.
$$

where

$$
\begin{gathered}
\langle x, x\rangle=\langle y, y\rangle=\langle x, \alpha\rangle=\langle y, \alpha\rangle=0, \quad\langle x, y\rangle=\langle\alpha, \alpha\rangle=1, \\
\alpha=x \times y, \quad \alpha \times x=x, \quad \alpha \times y=-y .
\end{gathered}
$$

Here in the sequel, $x, \alpha, y$ are also called the tangent, principal normal and binormal vector field of $r(s)$, respectively and the function $\kappa(s)$ is called the null curvature function of $r(s)$.

From (2.2) we have

$$
\kappa(s)=-\frac{1}{2}\left\langle r^{\prime \prime \prime}(s), r^{\prime \prime \prime}(s)\right\rangle
$$

and

$$
r^{(4)}(s)-2 \kappa(s) r^{\prime \prime}(s)-\kappa^{\prime}(s) r^{\prime}(s)=0 .
$$

\subsection{Representation formulas of null curves in $E_{1}^{3}$}

In [8], the authors defined the structure function $f$ of a cone curve in $Q^{2} \subset E_{1}^{3}$ and described it with $f$, where $Q^{2}=\left\{\left(x_{1}, x_{2}, x_{3}\right) \in E_{1}^{3}: x_{1}^{2}+x_{2}^{2}-x_{3}^{2}=0\right\}$. It is well-known that the integral curve of a cone curve in $Q^{2}$ is a null curve in $E_{1}^{3}$, so we can easily get the representation formulas of null curves in the sense of $[8]$.

Proposition $2.2([8])$. Let $r(s): I \rightarrow E_{1}^{3}$ be a null curve parameterized by null arc length parameter $s$. Then $r(s)$ can be written as

$$
r(s)=\int \frac{f}{2 f_{s}}\left(f-f^{-1}, 2, f+f^{-1}\right) d s,
$$

where $f(s)$ is called the structure function of $r(s)$. The structure function $f(s)$ and the null curvature function $\kappa(s)$ satisfy

$$
\kappa(s)=\frac{1}{2}\left[\left(\log f_{s}\right)_{s}\right]^{2}-\left[\left(\log f_{s}\right)_{s}\right]_{s}
$$

with $f_{s}=\frac{d f(s)}{d s}$. 


\subsection{Frenet formulas of space-like and time-like curves in $E_{1}^{3}$}

Let $r=r(s): I \rightarrow E_{1}^{3}$ be a space-like curve parameterized by arc length $s$ with the Frenet frame $\{T, N, B\}$.

Case 1 : If $\left\langle r^{\prime \prime}(s), r^{\prime \prime}(s)\right\rangle \neq 0$, the following Frenet equations are satisfied

$$
\left\{\begin{array}{l}
r^{\prime}(s)=T(s) \\
T^{\prime}(s)=\kappa(s) N(s) \\
N^{\prime}(s)=-\varepsilon \kappa(s) T(s)+\tau(s) B(s), \\
B^{\prime}(s)=\tau(s) N(s)
\end{array}\right.
$$

where $\langle T, T\rangle=1,\langle N, N\rangle=\varepsilon= \pm 1,\langle B, B\rangle=-\varepsilon,\langle T, N\rangle=\langle T, B\rangle=\langle B, N\rangle=$ 0 for some functions $\kappa$ and $\tau$ which are called the curvature and the torsion of $r$, respectively.

Case 2: If $\left\langle r^{\prime \prime}(s), r^{\prime \prime}(s)\right\rangle=0$, the Frenet equations are given by

$$
\left\{\begin{array}{l}
r^{\prime}(s)=T(s), \\
T^{\prime}(s)=N(s), \\
N^{\prime}(s)=\kappa(s) N(s), \\
B^{\prime}(s)=-T(s)-\kappa(s) B(s),
\end{array}\right.
$$

where $\langle T, T\rangle=\langle N, B\rangle=1,\langle N, N\rangle=\langle B, B\rangle=\langle T, N\rangle=\langle T, B\rangle=0$. The function $\kappa(s)$ is also called the curvature function of $r$.

Remark 2.3. Let $r$ be a space-like curve in Minkowski 3-space with arc length parameter $s$. In particular, the space-like curve $r$ is said to be null type spacelike if $\left\langle r^{\prime \prime}(s), r^{\prime \prime}(s)\right\rangle=0$.

If $r=r(s): I \rightarrow E_{1}^{3}$ is a time-like curve parameterized by arc length and framed by the Frenet frame $\{T, N, B\}$, then the following Frenet equations are satisfied

$$
\left\{\begin{array}{l}
r^{\prime}(s)=T(s) \\
T^{\prime}(s)=\kappa(s) N(s), \\
N^{\prime}(s)=\kappa(s) T(s)+\tau(s) B(s), \\
B^{\prime}(s)=-\tau(s) N(s),
\end{array}\right.
$$

where $\langle T, T\rangle=-1,\langle N, N\rangle=\langle B, B\rangle=1,\langle T, N\rangle=\langle T, B\rangle=\langle B, N\rangle=0$. Similarly to those of the space-like curve, the functions $\kappa(s)$ and $\tau(s)$ are called the curvature and the torsion of $r(s)$, respectively.

\subsection{Directional associated curves and self-associated curve of a null curve in $E_{1}^{3}$}

In this section, the definition of the directional associated curves of a null curve is given. Also, the self-associated curve of a null curve is defined in $E_{1}^{3}$.

Definition. Let $r(s): I \rightarrow E_{1}^{3}$ be a null curve parametrized by null arc length parameter $s$ with the Frenet frame $\{x, \alpha, y\}$ and $W$ a vector field along $r(s)$. A curve $\tilde{r}(\tilde{s}): I \rightarrow E_{1}^{3}$ is called the $W$-directional associated curve of $r(s)$ if $\tilde{r}(\tilde{s})$ is in the direction of $W$ which is in the Frenet frame of $\tilde{r}(\tilde{s})$ at each $r(s)$. 
Remark 2.4. From now on we assume that $\tilde{s}$ is the null arc length parameter if $\tilde{r}$ is a null curve and the arc length parameter if $\tilde{r}$ is a non-null curve.

Remark 2.5. If the vector filed $W$ is parallel to $x$ (respectively, $y$ or $\alpha$ ) the directional associated curve of $r(s)$ is called the $x$-directional (respectively, $y$-directional or $\alpha$-directional) associated curve and it can be expressed by, respectively,

$$
\begin{gathered}
\tilde{r}(\tilde{s}(s))=r(s)+\lambda(s) x(s), \\
\tilde{r}(\tilde{s}(s))=r(s)+\lambda(s) y(s)
\end{gathered}
$$

or

$$
\tilde{r}(\tilde{s}(s))=r(s)+\lambda(s) \alpha(s)
$$

for some function $\lambda$, which is called the distance function between $r(s)$ and $\tilde{r}(\tilde{s})$. The distance function $\lambda$ is assumed to be non-zero.

Example. Let $r(s)$ be a null curve of null curvature $-\frac{1}{2}$ and parametrized by null arc length given by

$$
r(s)=(\cos s, \sin s, s) .
$$

Then, the Frenet frame is given by

$x(s)=(-\sin s, \cos s, 1), \alpha(s)=(-\cos s,-\sin s, 0), y(s)=\frac{1}{2}(-\sin s, \cos s,-1)$.

Define $\tilde{r}$ by $\tilde{r}(s)=r(s)+2 \alpha(s)$. Then

$$
\tilde{r}(s)=(-\cos s,-\sin s, s) .
$$

Obviously, $\tilde{r}(s)$ is also a null curve parametrized by null arc length and its Frenet frame $\{\tilde{x}, \tilde{\alpha}, \tilde{y}\}$ is

$$
\tilde{x}(s)=(\sin s,-\cos s, 1), \tilde{\alpha}(s)=(\cos s, \sin s, 0), \tilde{y}(s)=\frac{1}{2}(\sin s,-\cos s,-1) .
$$

In this example, $\tilde{\alpha}=-\alpha$, so $\tilde{r}(s)$ is an $\alpha$-directional associated curve of $r(s)$.

Definition. Let $r(s): I \rightarrow E_{1}^{3}$ be a null curve parametrized by the null arc length parameter $s$ with the Frenet frame $\{x, \alpha, y\}$. The integral curve $\tilde{r}(\tilde{s})=\int y(\tilde{s}) d \tilde{s}$ is called the self-associated curve of $r(s)$.

\section{3. $x$-directional associated curves of a null curve in $E_{1}^{3}$}

Theorem 3.1. Let $r=r(s): I \rightarrow E_{1}^{3}$ be a null curve parametrized by null arc length parameter $s$ and $\tilde{r}(\tilde{s})$ its $x$-directional associated curve. Then, we get

(1) $\tilde{r}(\tilde{s})$ must be a null type space-like curve;

(2) the curvature function of $\tilde{r}(\tilde{s})$ satisfies

$$
\tilde{\kappa}= \pm \frac{1}{\lambda^{2}}
$$

(3) the null curvature function of $r(s)$ can be expressed by

$$
\kappa=\frac{1+4 \lambda^{\prime}+3 \lambda^{\prime 2}-2 \lambda \lambda^{\prime \prime}}{2 \lambda^{2}},
$$


where $\lambda(s)$ is the distance function.

Proof. The $x$-directional associated curve $\tilde{r}$ of $r$ is given by

$$
\tilde{r}(\tilde{s})=r(s)+\lambda(s) x(s),
$$

which implies

$$
\dot{\tilde{r}}(\tilde{s}) \frac{d \tilde{s}}{d s}=\left(1+\lambda^{\prime}\right) x+\lambda \alpha,
$$

where $\dot{\tilde{r}}(\tilde{s})=\frac{d \tilde{r}}{d \tilde{s}}$.

Then, (3.1) yields

$$
\langle\dot{\tilde{r}}, \dot{\tilde{r}}\rangle\left(\frac{d \tilde{s}}{d s}\right)^{2}=\lambda^{2} .
$$

Since the distance function $\lambda(s) \neq 0$ and $\tilde{r}$ is regular, $\tilde{r}(\tilde{s})$ must be a space-like curve, i.e., $\langle\dot{\tilde{r}}, \dot{\tilde{r}}\rangle>0$. By definition of the $x$-directional associated curve, we know $\tilde{r}(\tilde{s})$ must be a null type space-like curve, i.e., $\langle\ddot{\tilde{r}}, \ddot{\tilde{r}}\rangle=0$.

Let $\{\tilde{T}, \tilde{N}, \tilde{B}\}$ be the Frenet frame of $\tilde{r}$. Then $\dot{\tilde{r}}(\tilde{s})=\tilde{T}(\tilde{s})$. So, equation (3.1) can be written as

$$
\tilde{T} \frac{d \tilde{s}}{d s}=\left(1+\lambda^{\prime}\right) x+\lambda \alpha .
$$

Based on the definition of the $x$-directional associated curve and the Frenet frame of the null type space-like curve, we consider the following cases.

Case 1: $\tilde{N}(\tilde{s}(s)) \wedge x(s)=0$ along $r$.

In this case, we get

$$
\tilde{N}(\tilde{s}(s))=a(s) x(s)
$$

for some function $a(s) \neq 0$.

Differentiating (3.4) gives

$$
\tilde{\kappa} \tilde{N} \frac{d \tilde{s}}{d s}=a^{\prime} x+a \alpha
$$

from which,

$$
a(s)=0,
$$

a contradiction.

Case 2: $\tilde{B}(\tilde{s}(s)) \wedge x(s)=0$ along $r$.

We may suppose

$$
\tilde{B}(\tilde{s}(s))=a(s) x(s)
$$

for some function $a(s) \neq 0$.

Similarly as was given in Case 1, by differentiating (3.6), we have

$$
(-\tilde{T}-\tilde{\kappa} \tilde{B}) \frac{d \tilde{s}}{d s}=a^{\prime} x+a \alpha .
$$


Taking the scalar product on both sides of (3.7), we obtain

$$
\left(\frac{d \tilde{s}}{d s}\right)^{2}=a^{2} .
$$

By taking the scalar product with (3.7) with $\tilde{T}$ and using (3.3), we get

$$
-\left(\frac{d \tilde{s}}{d s}\right)^{2}=a \lambda \text {. }
$$

Thus, (3.8) and (3.9) give

$$
a=-\lambda \text {. }
$$

Therefore, we get $\frac{d \tilde{s}}{d s}= \pm \lambda$. Without loss of generality, we may assume

$$
\frac{d \tilde{s}}{d s}=\lambda
$$

Differentiating (3.3) with (3.10), we get

$$
\lambda^{\prime} \tilde{T}+\lambda^{2} \tilde{N}=\left(\lambda^{\prime \prime}+\lambda \kappa\right) x+\left(1+2 \lambda^{\prime}\right) \alpha-\lambda y,
$$

from which,

$$
\lambda^{\prime 2}=\left(1+2 \lambda^{\prime}\right)^{2}-2 \lambda\left(\lambda^{\prime \prime}+\lambda \kappa\right) .
$$

Thus, the null curvature function $\kappa$ can be expressed by

$$
\kappa=\frac{1+4 \lambda^{\prime}+3 \lambda^{\prime 2}-2 \lambda \lambda^{\prime \prime}}{2 \lambda^{2}} .
$$

Taking the scalar product with (3.7) and (3.11), we obtain

$$
\tilde{\kappa}=\frac{1}{\lambda^{2}}
$$

If $\frac{d \tilde{s}}{d s}=-\lambda$, equation (3.12) still holds with $\tilde{\kappa}=-\frac{1}{\lambda^{2}}$.

Corollary 3.2. Let $r(s): I \rightarrow E_{1}^{3}$ be a null curve with null arc length parameter $s$ and $\tilde{r}(\tilde{s})$ the $x$-directional associated curve. If the distance function $\lambda(s)$ is a nonzero constant, then we have

(1) the null curvature function of $r(s)$ is a positive constant and the curvature function of $\tilde{r}(\tilde{s})$ is a nonzero constant;

(2) $r(s)$ can be expressed as

$$
r(s)=C_{1} \sinh (\sqrt{2 \kappa}) s+C_{2} \cosh (\sqrt{2 \kappa}) s+C_{3} s,
$$

where $C_{1}, C_{2}, C_{3} \in E_{1}^{3}$;

(3) the $x$-directional associated curve $\tilde{r}(\tilde{s})$ is

$$
\tilde{r}(s)=C_{4} \sinh (\sqrt{2 \kappa}) s+C_{5} \cosh (\sqrt{2 \kappa}) s+C_{6} s,
$$

where $C_{4}, C_{5}, C_{6} \in E_{1}^{3}$. 
Proof. By Theorem 3.1, if the distance function is a nonzero constant, the null curvature function $\kappa$ is a positive constant and the curvature function $\tilde{\kappa}$ is a nonzero constant.

From (2.3), we have

$$
r^{(4)}=2 \kappa r^{\prime \prime} .
$$

Solving the equation (3.13), we get up to translation in $E_{1}^{3}$

$$
r(s)=C_{1} \sinh (\sqrt{2 \kappa}) s+C_{2} \cosh (\sqrt{2 \kappa}) s+C_{3} s .
$$

Therefore, the $x$-directional associated curve is given as

$$
\tilde{r}(s)=C_{4} \sinh (\sqrt{2 \kappa}) s+C_{5} \cosh (\sqrt{2 \kappa}) s+C_{6} s,
$$

where $C_{1}, C_{2}, C_{3}, C_{4}, C_{5}, C_{6} \in E_{1}^{3}$.

Corollary 3.3. Let $r(s): I \rightarrow E_{1}^{3}$ be a null curve parametrized by null arc length parameter $s$ and $\tilde{r}(\tilde{s})$ its $x$-directional associated curve. If the distance function $\lambda(s)$ is a linear function of $s$, then we obtain

(1) the null curvature function of $r(s)$ is

$$
\kappa(s)=a(s+b)^{-2},
$$

where $a \neq 0, b$ are constants;

(2) $r(s)$ can be expressed by one of the following forms:

(a) $r(s)=C_{1} s^{2}+C_{2} s^{(2+\sqrt{1+2 a})}+C_{3} s^{(2-\sqrt{1+2 a})}$ for $2 a>-1$,

(b) $r(s)=C_{1} s^{2}+C_{2} s^{2} \log s+C_{3} s^{2} \log ^{2} s$ for $2 a=-1$,

(c) $r(s)=C_{1} s^{2}+C_{2} s^{2} \sin [(\sqrt{-1-2 a}) \log s]+C_{3} s^{2} \cos [(\sqrt{-1-2 a}) \log s]$ for $2 a<-1$,

where $C_{1}, C_{2}, C_{3} \in E_{1}^{3}$;

(3) the curvature function of $\tilde{r}(\tilde{s})$ is given by

$$
\tilde{\kappa}(s)= \pm(c s+d)^{-2},
$$

where $c \neq 0, d$ are constants.

Proof. By Theorem 3.1, if the distance function is a linear function of $s$, the null curvature function can be written as

$$
\kappa(s)=a(s+b)^{-2},
$$

where $a \neq 0, b$ are constants. By a parameter transformation, we can put $b=0$.

From (2.3), we have

$$
s^{3} r^{(4)}-2 a s r^{\prime \prime}+2 a r^{\prime}=0 .
$$

Solving the differential equation (3.14), we get

(1) $r(s)=C_{1} s^{2}+C_{2} s^{(2+\sqrt{1+2 a})}+C_{3} s^{(2-\sqrt{1+2 a})}$ if $2 a>-1$,

(2) $r(s)=C_{1} s^{2}+C_{2} s^{2} \log s+C_{3} s^{2} \log ^{2} s$ if $2 a=-1$,

(3) $r(s)=C_{1} s^{2}+C_{2} s^{2} \sin [(\sqrt{-1-2 a}) \log s]+C_{3} s^{2} \cos [(\sqrt{-1-2 a}) \log s]$ if $2 a<-1$, 
where $C_{1}, C_{2}, C_{3} \in E_{1}^{3}$.

Also by Theorem 3.1, the curvature function of $\tilde{r}(\tilde{s})$ is given by

$$
\tilde{\kappa}(s)= \pm(c s+d)^{-2}
$$

where $c \neq 0, d$ are constants.

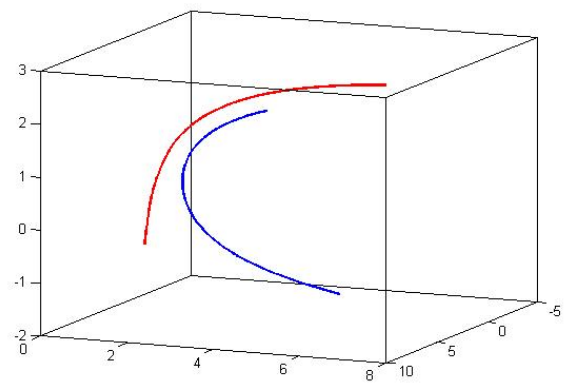

Figure 1. The blue curve is a given null curve and the red one is its $x$-directional associated curve when $\lambda=1, \kappa=1 / 2$.

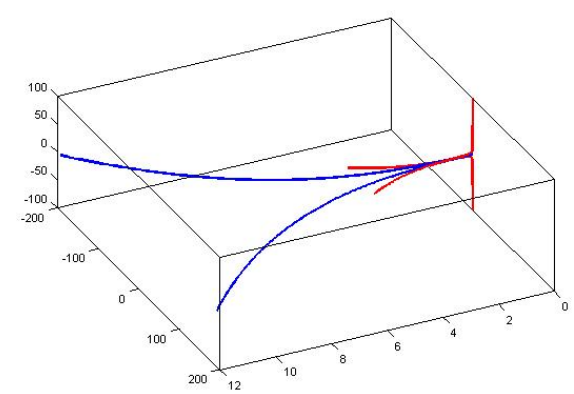

Figure 2. The red curve is a given null curve and the blue one is its $x$-directional associated curve when $\lambda=s, \kappa=4 / s^{2}$.

\section{4. $y$-directional associated curves of a null curve in $E_{1}^{3}$}

Theorem 4.1. Let $r=r(s): I \rightarrow E_{1}^{3}$ be a null curve parametrized by null arc length and $\tilde{r}(\tilde{s})$ its $y$-directional associated curve. Then we have

(1) the $y$-directional associated curve must be a null curve;

(2) the null curvature functions $\kappa$ and $\tilde{\kappa}$ satisfy

$$
\tilde{\kappa}=\kappa, \quad \kappa^{2}=2\left(\frac{1}{\lambda}\right)^{\prime}
$$


where $\lambda(s)$ is the distance function.

Proof. The definition of the $y$-directional associated curve of a null curve yields

$$
\tilde{r}(\tilde{s})=r(s)+\lambda(s) y(s),
$$

from which, we get

and

$$
\dot{\tilde{r}}(\tilde{s}) \frac{d \tilde{s}}{d s}=x+\lambda^{\prime} y-\lambda \kappa \alpha
$$

$$
\langle\dot{\tilde{r}}, \dot{\tilde{r}}\rangle\left(\frac{d \tilde{s}}{d s}\right)^{2}=\lambda^{2} \kappa^{2}+2 \lambda^{\prime} .
$$

It is not difficult to find that a null curve and a null type space-like curve in $E_{1}^{3}$ can be the $y$-directional associated curve of a null curve by the definition.

Case 1: If $\tilde{r}(\tilde{s})$ is a null curve with null arc length parameter $\tilde{s}$ and the Frenet frame $\{\tilde{x}, \tilde{\alpha}, \tilde{y}\}$, then we get $\dot{\tilde{r}}(\tilde{s})=\tilde{x}(\tilde{s})$ and

$$
\tilde{x}(\tilde{s}) \frac{d \tilde{s}}{d s}=x+\lambda^{\prime} y-\lambda \kappa \alpha .
$$

It implies

$$
\lambda^{2} \kappa^{2}+2 \lambda^{\prime}=0
$$

or, equivalently,

$$
\kappa^{2}=2\left(\frac{1}{\lambda}\right)^{\prime}
$$

Case 1.1: $\tilde{x}(\tilde{s}) \wedge y(s)=0$

Then, we get

$$
\tilde{x}(\tilde{s})=a(s) y(s)
$$

for some function $a(s) \neq 0$. Taking the scalar product with (4.2) and (4.4) implies $a(s)=0$, a contradiction.

Case 1.2: $\tilde{y}(\tilde{s}) \wedge y(s)=0$.

Similarly, we may put

$$
\tilde{y}(\tilde{s})=a(s) y(s)
$$

for some function $a(s) \neq 0$ and some parametrization $\tilde{s}=\tilde{s}(s)$.

Taking the scalar product with (4.2) and (4.5), we get

$$
\frac{d \tilde{s}}{d s}=a(s) .
$$

Thus, (4.2) implies

$$
\tilde{x} a=x+\lambda^{\prime} y-\lambda \kappa \alpha .
$$

Differentiating (4.6) yields

$$
a^{2} \tilde{\alpha}+\tilde{x} a^{\prime}=-\lambda \kappa^{2} x+\left(\lambda^{\prime \prime}+\lambda \kappa\right) y+\left(1-2 \lambda^{\prime} \kappa-\lambda \kappa^{\prime}\right) \alpha .
$$

Making use of (4.5) and (4.7), we get

$$
a^{\prime}=-a \lambda \kappa^{2} .
$$


Together with (4.3) and (4.8), we obtain

Thus, we have

$$
\frac{a^{\prime}}{a}=\frac{2 \lambda^{\prime}}{\lambda}
$$

$$
a=c \lambda^{2}
$$

for some positive constant $c$.

On the other hand, differentiating (4.5), we obtain

$$
-\tilde{\kappa} \tilde{\alpha} a=a^{\prime} y-a \kappa \alpha .
$$

Using (4.9) and (4.6), we get

$$
-a^{2} \tilde{\kappa} \tilde{x}=-a^{\prime} \alpha-a \kappa x+\left(a \kappa \lambda^{\prime}-\lambda \kappa a^{\prime}\right) y .
$$

Taking account of (4.5) and (4.10), we have

$$
\tilde{\kappa}=\kappa .
$$

Case 2: In case that $\tilde{r}(\tilde{s})$ is a null type space-like curve parametrized by arc length with the Frenet frame $\{\tilde{T}, \tilde{N}, \tilde{B}\}$, a contradiction is derived no matter the direction of $\tilde{N}$ or $\tilde{B}$ coincides with that of $y$.

Corollary 4.2. Let $r=r(s): I \rightarrow E_{1}^{3}$ be a null curve parametrized by null arc length $s$ and $\tilde{r}(\tilde{s})$ the $y$-directional associated curve. If the distance function $\lambda(s)$ is a nonzero constant, then we have

(1) $r(s)$ and $\tilde{r}(\tilde{s})$ are all null cubics;

(2) $r(s)$ can be expressed as

$$
r(s)=C_{1} s^{3}+C_{2} s^{2}+C_{3} s,
$$

where $C_{i} \in E_{1}^{3}(i=1,2,3)$.

Proof. In Theorem 4.1, if the distance function is a nonzero constant, both of the null curvatures $\kappa$ and $\tilde{\kappa}$ are equal to zero. Thus, they are null cubics.

From (2.3), we have

$$
r^{(4)}=0 .
$$

Therefore, up to translation, we get

$$
r(s)=C_{1} s^{3}+C_{2} s^{2}+C_{3} s,
$$

where $C_{1}, C_{2}, C_{3} \in E_{1}^{3}$. (For the null cubics, see [6].)

Corollary 4.3. Let $r(s): I \rightarrow E_{1}^{3}$ be a null curve with null arc length parameter $s$ and $\tilde{r}(\tilde{s})$ the $y$-directional associated curve. When the distance function $\lambda(s)$ is a linear function of $s$, we have

(1) the null curvature function is given by

$$
\tilde{\kappa}=\kappa=a(s+b)^{-1},
$$

where $a \neq 0, b$ are constants. 
(2) $r(s)$ can be expressed by

$$
r(s)=C_{1} \int u^{2}(s) d s+C_{2} \int u(s) v(s) d s+C_{3} \int v^{2}(s) d s,
$$

where $C_{1}, C_{2}, C_{3} \in E_{1}^{3}$.

Proof. In Theorem 4.1, if the distance function is a linear function of $s$, the null curvature functions are easily obtained as

$$
\tilde{\kappa}=\kappa=a(s+b)^{-1}
$$

where $a \neq 0, b$ are constants.

From (2.3), when $\kappa(s)=\frac{a}{s}$ (by a parameter transformation we can put $b=0)$, the curve $r(s)$ satisfies

$$
s^{2} r^{(4)}-2 a s r^{\prime \prime}+a r^{\prime}=0 .
$$

Solving the equation (4.13), we get

$$
r(s)=C_{1} \int u^{2}(s) d s+C_{2} \int u(s) v(s) d s+C_{3} \int v^{2}(s) d s
$$

for some functions $u$ and $v$ given by

$$
\left\{\begin{array}{l}
u(s)=\sqrt{s} J_{1}\left(\sqrt{-2 a} s^{\frac{1}{2}}\right), \\
v(s)=\sqrt{s} Y_{1}\left(\sqrt{-2 a} s^{\frac{1}{2}}\right) \quad \text { if } \quad a<0
\end{array}\right.
$$

and

$$
\left\{\begin{array}{l}
u(s)=\operatorname{Re}\left(\sqrt{s} Z_{1}\left(i \sqrt{2 a} s^{\frac{1}{2}}\right)\right), \\
v(s)=\operatorname{Im}\left(\sqrt{s} Z_{1}\left(i \sqrt{2 a} s^{\frac{1}{2}}\right)\right) \text { if } a>0,
\end{array}\right.
$$

where $C_{1}, C_{2}, C_{3} \in E_{1}^{3}, Z_{v}(s)$ is the cylinder function, $J_{v}(s)$ is the Bessel function of the first kind and $Y_{v}(s)$ is the Bessel function of the second kind (see $[9])$.

\section{5. $\alpha$-directional associated curves of a null curve in $E_{1}^{3}$}

Theorem 5.1. Let $r: I \rightarrow E_{1}^{3}$ be a null curve parametrized by null arc length parameter $s$ and $\tilde{r}(\tilde{s})$ its $\alpha$-directional associated curve. When the direction of the principal normal vector field of $\tilde{r}(\tilde{s})$ coincides with that of $\alpha$, we have

1. $\tilde{r}(\tilde{s})$ is a null curve written as

$$
\tilde{r}(s)=\lambda \int y(s) d s,
$$

that is, $r(s)$ is the binormal donor curve of $\tilde{r}(\tilde{s})$, where $\lambda$ is a nonzero constant. In this case, the null curvature function $\kappa$ of $r(s)$ is a nonzero constant and $r(s)$ can be expressed by one of following forms:

(1) $r(s)=C_{1} \sinh (\sqrt{2 \kappa}) s+C_{2} \cosh (\sqrt{2 \kappa}) s+C_{3} s$ for $\kappa>0$,

(2) $r(s)=C_{1} \sin (\sqrt{-2 \kappa}) s+C_{2} \cos (\sqrt{-2 \kappa}) s+C_{3} s$ for $\kappa<0$, 
where $C_{1}, C_{2}, C_{3} \in E_{1}^{3}$.

2. $\tilde{r}(\tilde{s})$ is a curve on a de Sitter 3-space or hyperbolic 3-space written as

$$
\tilde{r}(s)=r(s)+C \alpha(s)
$$

for some nonzero constant $C$. In this case, the null curvature function $\kappa$ of $r(s)$ is a constant and $r(s)$ is given by

(1) $r(s)=C_{1} s^{3}+C_{2} s^{2}+C_{3} s$ for $\kappa=0$;

(2) $r(s)=C_{1} \sinh (\sqrt{2 \kappa}) s+C_{2} \cosh (\sqrt{2 \kappa}) s+C_{3} s$ for $\kappa>0$;

(3) $r(s)=C_{1} \sin (\sqrt{-2 \kappa}) s+C_{2} \cos (\sqrt{-2 \kappa}) s+C_{3} s$ for $\kappa<0$,

where $C_{1}, C_{2}, C_{3} \in E_{1}^{3}$.

3. When $\tilde{r}(\tilde{s})$ is not a null curve or a curve on a de Sitter 3-space or hyperbolic 3-space, it is given by

$$
\tilde{r}(s)=r(s)-\frac{2}{\kappa+C} \alpha(s) .
$$

In this case, the null curvature function $\kappa(s)$ of $r(s)$ is given by the differential equation

$$
2 \kappa^{\prime \prime}-3 \kappa^{2}-2 \kappa C+C^{2}=0,
$$

where $C$ is a constant.

Proof. In the proof, we use the same parameter $s$ for the null curve $r$ and the associated curve $\tilde{r}$. Then, we have

$$
\tilde{r}(s)=r(s)+\lambda(s) \alpha(s),
$$

from which,

$$
\begin{gathered}
\tilde{r}^{\prime}(s)=(1+\lambda \kappa) x+\lambda^{\prime} \alpha-\lambda y, \\
\tilde{r}^{\prime \prime}(s)=\left(2 \lambda^{\prime} \kappa+\lambda \kappa^{\prime}\right) x+\left(1+2 \lambda \kappa+\lambda^{\prime \prime}\right) \alpha-2 \lambda^{\prime} y .
\end{gathered}
$$

It implies

$$
\left\langle\tilde{r}^{\prime}, \tilde{r}^{\prime}\right\rangle=\lambda^{\prime 2}-2 \lambda-2 \lambda^{2} \kappa .
$$

From (2.1) and (5.1), we have

$$
\left(\tilde{r}^{\prime} \times \tilde{r}^{\prime \prime}\right) \times \tilde{r}^{\prime}=\left(\lambda^{\prime} \lambda^{\prime \prime}-\lambda^{\prime}-2 \lambda \lambda^{\prime} \kappa-\lambda^{2} \kappa^{\prime}\right) \tilde{r}^{\prime}-\left(\lambda^{\prime 2}-2 \lambda-2 \lambda^{2} \kappa\right) \tilde{r}^{\prime \prime} .
$$

When the direction of the principal normal vector field of $\tilde{r}(\tilde{s})$ coincides with that of $\alpha$, from (5.3), we have

$$
\left\{\begin{array}{l}
2 \lambda^{\prime}\left(\lambda^{\prime 2}-2 \lambda-2 \lambda^{2} \kappa\right)=\lambda\left(\lambda^{\prime} \lambda^{\prime \prime}-\lambda^{\prime}-2 \lambda \lambda^{\prime} \kappa-\lambda^{2} \kappa^{\prime}\right), \\
\left(\lambda^{\prime 2}-2 \lambda-2 \lambda^{2} \kappa\right)\left(2 \lambda^{\prime} \kappa+\lambda \kappa^{\prime}\right)=(1+\lambda \kappa)\left(\lambda^{\prime} \lambda^{\prime \prime}-\lambda^{\prime}-2 \lambda \lambda^{\prime} \kappa-\lambda^{2} \kappa^{\prime}\right) .
\end{array}\right.
$$

We put

$$
A=\lambda^{\prime 2}-2 \lambda-2 \lambda^{2} \kappa, \quad B=\frac{1}{2} A^{\prime}=\lambda^{\prime} \lambda^{\prime \prime}-\lambda^{\prime}-2 \lambda \lambda^{\prime} \kappa-\lambda^{2} \kappa^{\prime} .
$$

Case 1: $A \equiv 0$. 
Since $\lambda \neq 0, B=0$. From (5.2), $\tilde{r}(s)$ is a null curve. From the Frenent frame $(2.2), \tilde{r}(s)$ can be written as

$$
\tilde{r}(s)=c \int y(s) d s,
$$

where $c$ is a nonzero constant.

Thus, from (5.5) and (5.1), $\lambda$ and $\kappa$ are non-zero constants. Then $r(s)$ can be written as

(1) $r(s)=C_{1} \sinh (\sqrt{2 \kappa}) s+C_{2} \cosh (\sqrt{2 \kappa}) s+C_{3} s$ for $\kappa>0$;

(2) $r(s)=C_{1} \sin (\sqrt{-2 \kappa}) s+C_{2} \cos (\sqrt{-2 \kappa}) s+C_{3} s$ for $\kappa<0$,

where $C_{1}, C_{2}, C_{3} \in E_{1}^{3}$.

Case 2: $A \neq 0, B=0$.

From (5.4), $\lambda$ is a nonzero constant and $\kappa^{\prime}=0$. Therefore, $r(s)$ is given as follow:

(1) $r(s)=C_{1} s^{3}+C_{2} s^{2}+C_{3} s$ for $\kappa=0$;

(2) $r(s)=C_{1} \sinh (\sqrt{2 \kappa}) s+C_{2} \cosh (\sqrt{2 \kappa}) s+C_{3} s$ for $\kappa>0$;

(3) $r(s)=C_{1} \sin (\sqrt{-2 \kappa}) s+C_{2} \cos (\sqrt{-2 \kappa}) s+C_{3} s$ for $\kappa<0$,

where $C_{1}, C_{2}, C_{3} \in E_{1}^{3}$.

Its $\alpha$-directional associated curve is expressed as

$$
\tilde{r}(s)=r(s)+\lambda \alpha(s),
$$

where $\lambda$ is a nonzero constant. Apparently, $\tilde{r}(s)$ is a curve on a de Sitter 3-space or a hyperbolic 3 -space.

Case 3: $A \neq 0, B \neq 0$.

From (5.4), we have

$$
\frac{2 \lambda^{\prime} A}{A\left(2 \lambda^{\prime} \kappa+\lambda \kappa^{\prime}\right)}=\frac{\lambda B}{(1+\lambda \kappa) B}
$$

i.e.,

$$
2 \lambda^{\prime}=\lambda^{2} \kappa^{\prime}
$$

Solving the differential equation (5.6), we have

$$
\lambda=\frac{-2}{\kappa+C},
$$

where $C$ is a constant of integration.

Substituting (5.7) into (5.4) yields

$$
2 \kappa^{\prime \prime}-3 \kappa^{2}-2 \kappa C+C^{2}=0 .
$$

Solving (5.8) (by a parameter transformation), we have the solution in parametric forms:

$$
\left\{\begin{array}{l}
s= \pm \frac{1}{\sqrt{|2 C|}} \int\left(C_{1}+\eta^{3} \pm \eta^{2}\right)^{\frac{-1}{2}} d \eta+C_{2} \\
\kappa= \pm 2 C \eta-C
\end{array}\right.
$$


or

$$
\left\{\begin{array}{l}
s= \pm \frac{1}{\sqrt{|2 C|}} \int\left(C_{1}-\eta^{3} \pm \eta^{2}\right)^{\frac{-1}{2}} d \eta+C_{2} \\
\kappa= \pm 2 C \eta-C
\end{array}\right.
$$

where $C, C_{1}, C_{2}$ are constants (see [9]).

In this case, its $\alpha$-directional associated curve can be written as

$$
\tilde{r}(s)=r(s)-\frac{2}{\kappa+C} \alpha(s),
$$

where $C$ is a constant and $\alpha(s)=r^{\prime \prime}(s)$.

Theorem 5.2. Let $r: I \rightarrow E_{1}^{3}$ be a null curve parametrized by null arc length parameter $s$ and $\tilde{r}(\tilde{s})$ the $\alpha$-directional associated curve. When the direction of the binormal vector field of $\tilde{r}(\tilde{s})$ coincides with that of $\alpha$, we have

1. $\tilde{r}(\tilde{s})$ is a curve on a de Sitter 3-space or hyperbolic 3-space written as

$$
\tilde{r}(s)=r(s)+C \alpha(s)
$$

for some non-zero constant $C$. In this case, the null curvature function of $r(s)$ is a non-zero constant and $r(s)$ is given by

(1) $r(s)=C_{1} \sinh (\sqrt{2 \kappa}) s+C_{2} \cosh (\sqrt{2 \kappa}) s+C_{3} s$ for $\kappa>0$;

(2) $r(s)=C_{1} \sin (\sqrt{-2 \kappa}) s+C_{2} \cos (\sqrt{-2 \kappa}) s+C_{3} s$ for $\kappa<0$, where $C_{1}, C_{2}, C_{3} \in E_{1}^{3}$.

2. When $\tilde{r}(\tilde{s})$ is not a curve on a de Sitter 3-space or hyperbolic 3-space, it is given by

$$
\tilde{r}(s)=r(s)-\frac{2}{\kappa+C} \alpha(s) .
$$

In this case, the null curvature function $\kappa(s)$ of $r(s)$ is given by the differential equation

$$
2 \kappa^{\prime \prime}-3 \kappa^{2}-2 \kappa C+C^{2}=0
$$

where $C$ is a constant.

Remark 5.3. For a null curve $r$ with the $\alpha$-directional associated curve $\tilde{r}$, if the binormal vector field of $\tilde{r}$ is parallel to $\alpha$ or the principal normal vector field of $\tilde{r}$ is parallel to $\alpha$, we have similar results.

\section{Self-associated curve of a null curve in $E_{1}^{3}$}

Theorem 6.1. Let $r(s)$ be a null curve in Minkowski 3-space $E_{1}^{3}$ with null arc length parameter $s$ and $\tilde{r}(\tilde{s})$ its self-associated null curve. Then the followings are equivalent:

(1) The null curvature functions $\kappa$ and $\tilde{\kappa}$ satisfy $\tilde{\kappa}=\frac{1}{\kappa}$.

(2) The self-associated curve of $\tilde{r}(\tilde{s})$ is $r(s)$.

(3) $r(s)$ is the binormal donor curve of $\tilde{r}(\tilde{s})$. 
Proof. Define $\tilde{r}(\tilde{s})=\int y(\tilde{s}) d \tilde{s}$, where $\tilde{s}$ denotes the null arc length parameter of $\tilde{r}$. Let $\{\tilde{x}, \tilde{\alpha}, \tilde{y}\}$ be the Frenet frame of $\tilde{r}$.

From $\tilde{r}(\tilde{s})=\int y(\tilde{s}) d \tilde{s}$, we have

$$
\tilde{x}(\tilde{s})=y(\tilde{s}) .
$$

Differentiating (6.1) with respect to $\tilde{s}$, we have

$$
\tilde{\alpha}=-\kappa \alpha \frac{d s}{d \tilde{s}}
$$

It gives

$$
\left(\frac{d \tilde{s}}{d s}\right)^{2}=\kappa^{2}
$$

For convenience, we put

$$
\frac{d \tilde{s}}{d s}=-\kappa
$$

Then, we get

$$
\tilde{\alpha}=\alpha .
$$

Differentiating (6.2), we have

$$
\tilde{\kappa} \tilde{x}-\tilde{y}=(\kappa x-y) \frac{d s}{d \tilde{s}},
$$

in other words,

$$
\tilde{\kappa} \tilde{x}-\tilde{y}=-x+\frac{y}{\kappa} .
$$

It implies

$$
\tilde{\kappa}=\frac{1}{\kappa} .
$$

Therefore, we get

$$
\tilde{y}(\tilde{s})=x(s),
$$

from which,

$$
\int \tilde{y}(\tilde{s}) d s=\int x(s) d s=r(s) .
$$

This completes the proof.

Finally, we give a simple example to show the null curve and its self-associated curve. Let $r(s)=(\cos s, \sin s, s)$ with null curvature $\kappa=-1 / 2$ and parametrized by null arc length. 


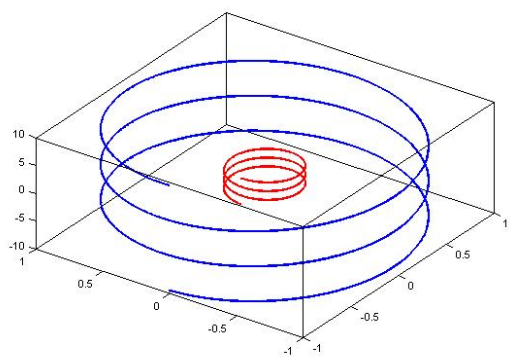

FiguRE 3 . The blue curve is a null curve and the red one is its self-associated curve.

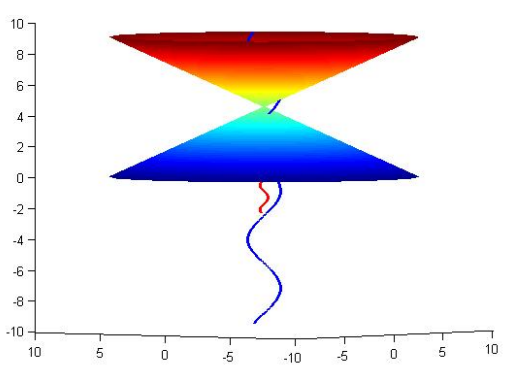

FIGURE 4. A null curve and its self-associated null curve on light-like cone.

\section{References}

[1] W. B. Bonnor, Null curves in a Minkowski space-time, Tensor (N.S.) 20 (1969), 229-242.

[2] B. Y. Chen, When does the position vector of a space curve always lie in its rectifying plane, Amer. Math. Monthly 110 (2003), no. 2, 147-152.

[3] J. H. Choi, T. H. Kang, and Y. H. Kim, Bertrand curves in 3-dimensional space forms, Appl. Math. Comput. 219 (2012), no. 3, 1040-1046.

[4] J. H. Choi and Y. H. Kim, Associated curves of a Frenet curve and their applications, Appl. Math. Comp. 218 (2012), no. 18, 9116-9124.

[5] _ Note on null helices in $E_{1}^{3}$, Bull. Korean Math. Soc. 50 (2013), no. 3, 885-899.

[6] J. Inoguchi and S. Lee, Null curves in Monkowski 3-space, Int. Electron. J. Geom. (2008), no. $2,40-83$.

[7] H. Liu, Curves in the lightlike cone, Beiträge Algebra Geom. 45 (2004), no. 1, 291-303.

[8] H. Liu and Q. Meng, Representation formulas of curves in a two- and three-dimensional lightlike cone, Results Math. 59 (2011), no. 3-4, 437-451.

[9] A. D. Polyanin and V. F. Zaitsev, Handbook of Exact Solutions for Ordinary Differential Equations, 2nd edition, Chapman and Hall/CRC, 2003. 
JINHUA QIAN

Department of Mathematics

Northeastern University

Shenyang 110004, P. R. China

E-mail address: ruoyunqian@163.com

Young Ho Kim

Department of Mathematics

KyungPoOK National University

DAEgu 702-701, Korea

E-mail address: yhkim@knu.ac.kr 